\title{
The influence of the structure of laminar flows on the characteristics of equipment
}

\author{
Vasyl Arsirii ${ }^{1, *}$, Oleg Kravchenko ${ }^{2}$, Bohdan Savchuk ${ }^{3}$, and Olena Arsirii ${ }^{4}$ \\ ${ }^{1,4}$ Odessa State Polytechnic University, 65044 Shevchenko av. 1, Ukraine \\ ${ }^{2}$ National University "Odessa Maritime Academy" 8, Didrikhson str., Odessa,65029, Ukraine \\ ${ }^{3}$ Odessa State Academy of Building and Architecture, 65029 Didrichson str 4, Ukraine
}

\begin{abstract}
The new method of visual diagnostics of liquid motion processes in physical models showed a high degree of the flow structure organization. Visual pictures made it possible to develop a hydraulic experiment to reveal the dimensions of the transverse structure in the form of layers and zones of flow separation from the channel walls. Visual diagnostics is the basis for comprehensive equipment design. Visual studies of the flow structure provide information for improving equipment by changing the geometry of the flow paths. Hydraulic studies show the change in the resistance of the equipment channels. Based on the results of visual and hydraulic studies, the wave character of the distribution of the pulsation velocity components was revealed. The regularities of the velocity distribution allow predicting the minimum or maximum values of the resistances of the flow paths of the equipment.

Keywords: Visualization; flow structures; laminar mode; resistance.
\end{abstract}

\section{Introduction}

The main task of mechanics of liquids and gases is to calculate the hydrodynamic parameters of the operation of various devices. Studying the patterns of flow organization in technological systems is the key to solving many problems. Despite the fact that this science has been forming for more than a century, Feynman's lectures on physics formulated an urgent problem in the design of equipment: "There is a very old physical problem common to many branches of activity and science. This is not a problem of searching for new elementary particles ... in its simplest form. There is a problem of what pressure $P$ needed to move a given amount of water through the pipe? And no one based only on the laws and on the properties of water itself, does not know

\footnotetext{
* Corresponding author: vasily.arsiry@gmail.com
} 
how to answer this question "[1]. That is, the imperfection of theoretical models and laws of hydrodynamics is one of the most pressing problems of technical progress. For example, energy consumption for compression and movement of deformable media is $5-15 \%$ in power engineering. Therefore, the main task of the models of fluid and gas mechanics is to offer the designer methods for improving hydro- and aerosystems to reduce resistance and accordingly increase the amount of transported water at a given initial pressure. Modern ideas about the movement of liquids and gases are based on the concept of turbulence [2]. The equations describing the turbulent flow provide only the averaged parameters of speed and pressure. The main cause of these problems is the optical transparency of liquids and gases (water, air, oil and gas), so their flow structure is not available for study [3, 4].

The visual diagnosis of the flow is especially important in the visualization and analysis of the dynamic processes. Therefore, an important task is the development of informative visualization methods, as well as methods for analyzing visual images to decipher the distribution fields of real hydrodynamic parameters. The study of the structure in the flow parts can be the key to solving many problems of various kinds in both technological and biological systems $[5,6]$.

Object of the article: presentation of a complex method for solving problems of optimization of hydrodynamic parameters of devices, which includes two stages. The first stage is the visualization of flows for the structure during the movement of liquids and gases. The second stage is optimization of the geometry of the flow paths to improve the hydraulic devices of the devices.

\section{Well-known models of hydrodynamic processes}

The hydrodynamic models are based on the results of a large number of scientific researches. Most often, the dependences of the dynamic indicators (flow rate) on the initial potentials (pressure) are studied with average value of their pulsation components. Several important experiments can be allocated for the presentation of modern models of the dynamic processes of liquids and gases.

D. Mendeleev in 1880 determined the regularities of calculating pressure losses in the flow paths to overcome resistance: at low speeds, they increase linearly with speed; at high speeds, losses are proportional to the square of the speed. The idea is also formulated that the main processes occur at the boundary layer and a model of roughness was proposed. Difficulties in obtaining information about processes within flows and uncertainties in measuring the parameters of fluid motion have led to the creation of models of flow continuity and averaging of parameters. Most of the studies are still related to the processes of separation of flows from the walls of channels [7]. However, it was the complex experiments of Reynolds (1883), where visual and hydraulic studies were combined, that became the most productive for understanding the dynamic processes of fluids. Visual studies made it possible to record two fundamentally different modes of movement. At low velocities, the introduction of a dye into the flow does not change the jet thickness, which indicates minimal diffusion [8]. That is, adjacent layers do not mix at low speeds. This flow mode was called laminar. With an increase in the flow rate, a mode occurs when the dye is rapidly mixed. 
Such a mode is called turbulent or chaotic. Comprehensive Reynolds studies made it possible to develop a well-predicted value of the critical Reynolds number $R_{c r}=2320$, which characterizes the transition from a layered flow to a chaotic one. Only after Reynolds comprehensive research it became possible to investigate and correctly represent the values of resistances $R$ for calculating pressure losses in channels at different flow mode. Poiseuille's experiments made it possible to develop a formula for calculating the resistance values for the laminar mode.

$$
R=64 / R e
$$

The Poiseuille's on the Nikuradze diagram (1933) curve shows the decrease in resistances $R$ with an increase in the Reynolds number in the range $R e<2320$. Blasius's experiments made it possible to develop a formula for calculating the values of resistance $R$ in channels with smooth walls (roughness $\Delta=0$ ) for a turbulent mode.

$$
R=0,3164 / R e^{0,25}
$$

On Nikuradse diagram, the Blasius curve is also one line of decrease in $R$ in the range of Reynolds numbers $\operatorname{Re}>2320$.

\section{Method of flow structure visual diagnostics}

The article presents a new method for improving the parameters of power equipment operation by visualizing the processes of fluid movement in special physical models. The method of the flows structure visual diagnostics (FSVD) makes it possible to reveal the structure of flows of liquids or gases not only at the walls arias of the channels, but also the layers inside the flow, that are not mixed [8]. New knowledge about the structure of flows makes it possible to improve the geometry of the channels to improve the energy performance of equipment by reducing the resistance of the flow paths.

Method FSVD can be used as the first stage of designing new or improving known flow parts of equipment. The visualization of flows is carried out in special physical models with the characteristic dimensions of the flow paths. An optically active liquid (based on vanadium oxide $V_{2} O_{5}$ ) moves in the model. The speed of movement is consistent with the speed in a real device according to the Reynolds number. Elongated vanadium oxide molecules are oriented in the flow in accordance with velocity gradients. The stresses in the flow associated with the velocity gradients in polarized light are reflected by the change in the intensity of the illumination of the flow pattern in the entire field of the model. Light areas indicate positive velocity gradients, dark areas show negative gradients. Thus, visualization characterizes the field or the complete picture of instantaneous velocity values. Figure 1 shows the result of visual studies of liquid or gas flow in a $90^{\circ}$ turn. 


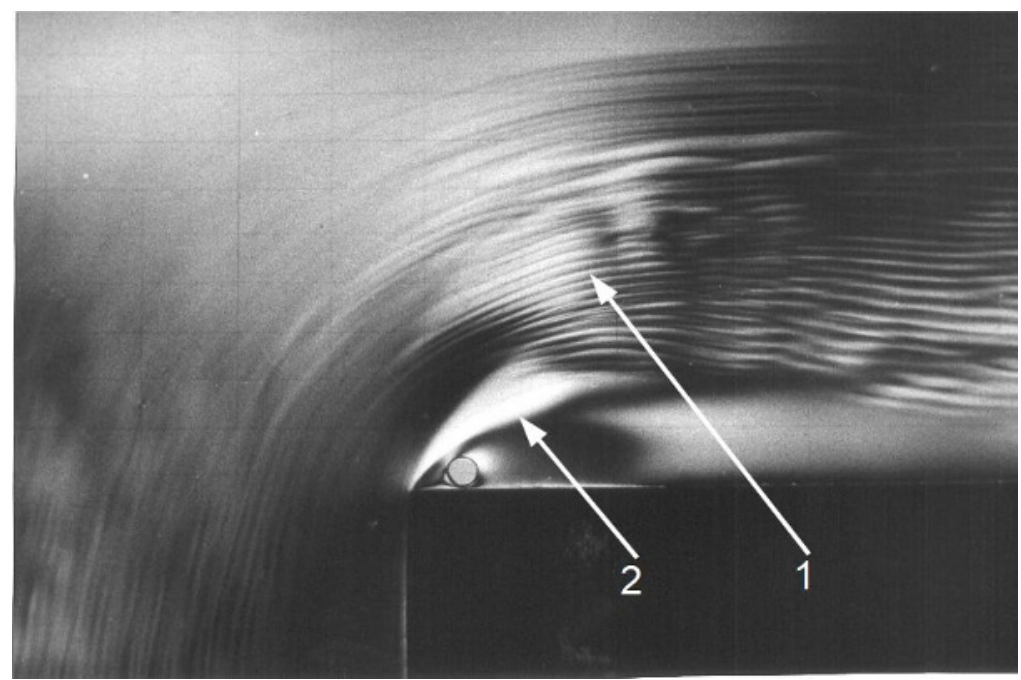

1 - Lines in the channel cross-section that have a stable structure; 2 - The boundaries of flow separation from the channel walls.

Fig. 1. Image of the structure of flow in a $90^{\circ}$ turn.

The visualization revealed lines in the channel cross-section that have a stable structure. In the figure, one can clearly distinguish also the boundaries of flow separation from the channel walls. Visual diagnostic of flows can be considered the first stage of design. In the second stage, you can improve the geometry of the channels to optimize the energy parameters of the equipment. At the second stage, you can improve the geometry of the channels to optimize the energy parameters of the equipment. To reduce the resistance of the flow paths, it is sufficient to eliminate the dissipative zones by replacing them with inserts, as well as by optimizing the placement of the identified layers.

\section{Visual studies of the laminar flow structure}

The experience of visual studies of flows in physical models shows that the movement of fluids under certain combinations of geometric and hydraulic parameters has a stable structure both in the transverse and longitudinal sections. The article presents the results of studies of transverse flow structures only. In fig. 2 shows the results of visual diagnostics of the structure of a laminar jet in a flooded space. A physics experiment using the FSVD method at low Reynolds numbers shows layers that have minimal diffusion and therefore a stable cross-sectional structure. 


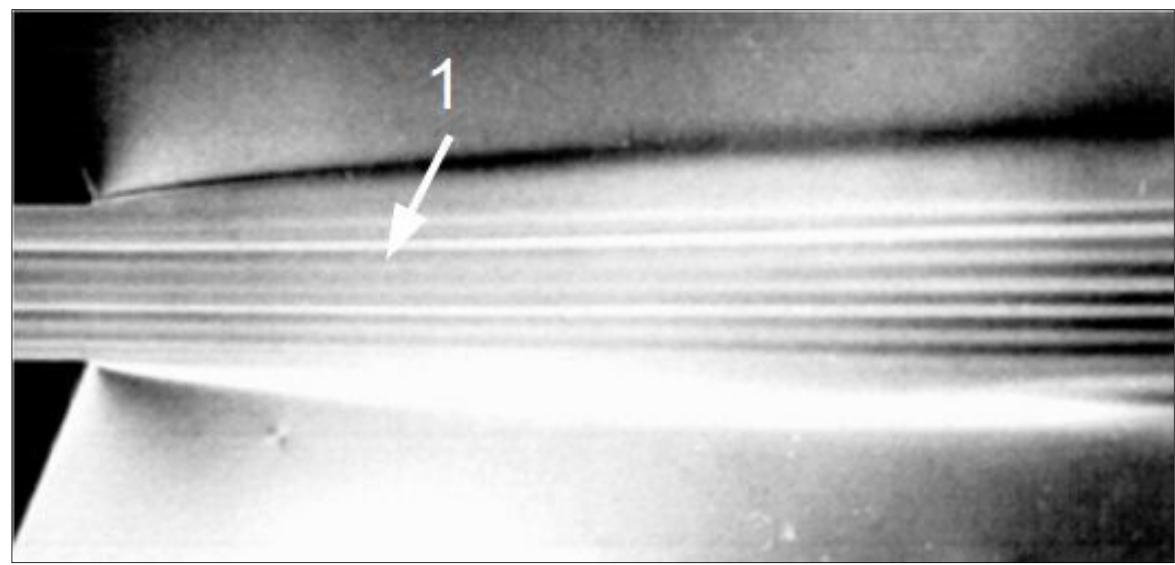

a.



b.

a) the structure of the laminar stream $(\mathrm{Re}=1700)$

b) the structure of the laminar stream $(\mathrm{Re}=2100)$

1 - transverse structures (streamlines);

Fig. 2. Image of the structure of a laminar stream.

Light and dark stripes are clearly visible in the laminar jet image. Stable dimensions made it possible to determine the frequency of changes in illumination. The distance between the peaks or extrema of the illumination of the light stripes (or dark stripes) is $\lambda=0.52 \mathrm{~mm}$. Thus, $\lambda$ is the wavelength of the periodic nature of the distribution of the revealed structure of the illumination intensity.

\section{Hydraulic studies of laminar flow structure}

Visual pictures of the stable nature of the structure of the flow of a laminar flow made it possible to develop and conduct a series of hydraulic experiments. The hydraulic experiment is the second stage in the study of the structure of the laminar flow. It is 
necessary to answer whether there is a correspondence (correlation) between the visual patterns of the flow structure and the change in velocity in the cross section of the laminar flow. That is, the size of the wave character of the distribution of the optical density of the bands from light to dark and again to light correspond to the dimensions of the wave character of the change in the real velocity of the flow layers in the cross section at a given constant initial pressure $\mathrm{P}_{1}$.

For the hydraulic experiment, 8 models in the form of thin sheets of getinax of different thickness were made simultaneously on the machine (bench). A channel was cut in all sheets, in which the length 1 and width $b$ remained constant. In the experiments, only the channel height $h$ was changed. (Fig. 3). Twelve experiments were carried out in which one or several models were clamped between two absolutely smooth plates with water inlet and outlet manifolds.

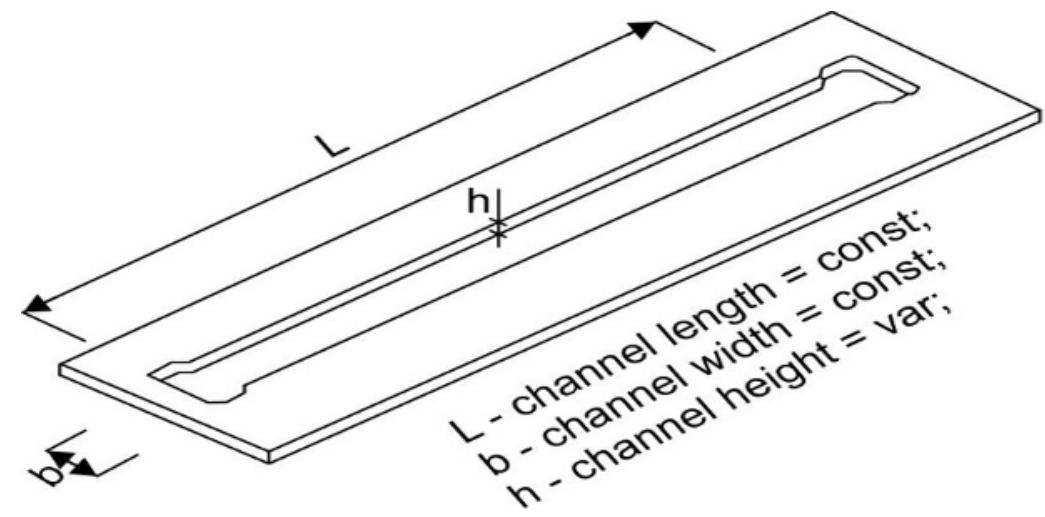

Fig. 3. Model for study of dependence $\lambda=\mathrm{f}(\operatorname{Re}, h)$

In each experiment, the pressure $P_{l}$ was varied discretely in the inlet manifold. In this case, two parameters were measured: pressure $P_{1}$ and water flow rate to calculate the velocity $V$ in the channel. The measured parameters of velocity and pressure made it possible to calculate the resistant coefficients $R$ in 12 variants of straight channels with a change in height in the range $h=0.9 \div 2.55 \mathrm{~mm}$, as well as a change in the Reynolds number Re of each individual experiment. The results of hydraulic experiments are shown in Fig. 4 in the form of 12 dependences of the channel resistances on the Reynolds number $\lambda=f(R e)$.

The walls of the channels had a smooth surface (roughness $\Delta=0 \mathrm{~mm}$ ); therefore, the results obtained can be compared with the Poiseuille's and Blasius's formulas. Deviations of the hydraulic friction coefficient $R$ from the values calculated by the Blasius formula (at $R e>2500$ ) are $\Delta R= \pm 16 \%$. At $R e<2320$, the deviations of the resistances from the values calculated by the Poiseuille formula are more than $\Delta R= \pm$ $20 \%$.

Analysis of the parameters of hydraulic experiments showed that a linear change in the height of the channels in the range $h=0.9 \div 2.55 \mathrm{~mm}$ corresponds to the wave nature of the change in the resistance $\mathrm{R}$ of the channels.. 


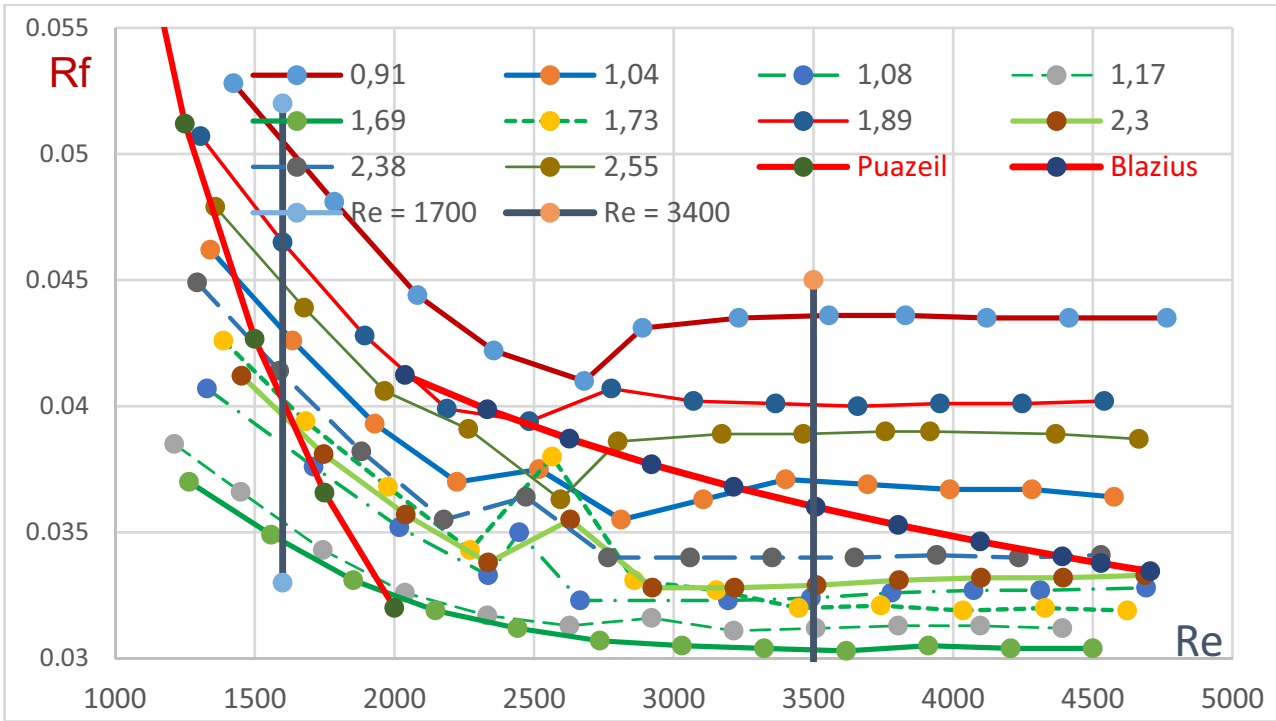

Fig. 4. Results of the study of the dependence $R=f(R e, h)$.

The wavelength of the resistance changes $\lambda_{R}$ coincided with the value of the wavelength $\lambda_{L}$ of the change in the optical density of the bands in the visual images of the laminar flow structure in Fig. 2. The analysis of the experimental results showed that for a given value of the Reynolds number $(R e=$ const), the linear change in the channel height $\mathrm{h}[\mathrm{mm}]$ corresponds to the wave character of the change in resistance with a wavelength $\lambda_{R}=0.52 \mathrm{~mm}$.

The results of hydraulic experiments are in good correlate with the wave character of the change in the optical density of the bands in the visual images of the laminar flow structure in Fig. 2 with a wavelength $\lambda_{L}=0.52 \mathrm{~mm}$. The results presented in Figure 2 eliminate the problem of measurement uncertainty in many laminar flow studies. However, the resistance $R$ is only a coefficient of proportionality between the main parameters of the movement of liquids: pressure $P$ and speed $V$. Therefore, the results obtained are best interpreted as follows. For the range of channel transverse dimensions $\mathrm{h}=0.91 \div 2.55 \mathrm{~mm}$, it is possible to determine the channel dimensions in which, at a given initial pressure $\mathrm{P} 1$, the velocity value will be greater than the average velocity calculated by the Poiseuille formulas (laminar mode) or Blasius formulas (turbulent mode). To determine the size of the channel, where the speed will be higher than the average, you can use the formula 3:

$$
b_{V \max }=n \lambda+0.25 \lambda
$$

$n$ - multiple of wavelengths $\lambda$. 
If necessary, you can solve the inverse problem of ensuring maximum resistance, for example, when designing the dimensions of seals. In this case, the channel size can be calculated using the formula 4 :

$$
b_{V \min }=n \lambda+0.75 \lambda
$$

The patterns distribution of transverse structures can be used in the design of flow paths for the movement of liquids and gases. An odd number of half-waves of pulsating velocity components $\Delta V$ corresponds to the positive influence of transverse structures; an even number of half-waves corresponds to the dissipative effect of the distribution of the velocity components and generates the maximum flow pulsations at the channel wall.

The results of hydraulic experiments have confirmed the presence of a flows structure that have a positive or negative effect on the speed of movement of liquids or gases. However, it is easiest to identify the flows structure by visual research. The results of visual and hydraulic experiments by A. Losievsky (1933) can be called a similar method for improving flow paths or channels. Layers or transverse structures of river flows were identified visually. Models explaining the nature of the transverse flow structures allowed M. Velikanov (1936) to put forward a hypothesis that the internal flow structure is inherent in any motion of liquids and gases [12]. Experience in improving various power equipment over the past 20 years [8] showed the promise of designing flow paths with an additional stage of visualization of the flow structure. However, the flow structure model is still little used in the design of technical devices.

\section{Conclusions}

The designing of equipment, the operation of which is associated with the movement of liquids and gases, has a number of problems. Often, the real productivity of the equipment is less than that calculated by the known formulas. The negative phenomena are noise, vibration.

The main reason for the difficulties in eliminating problems and negative phenomena is the lack of informative research methods for studying flows. It is the physical visual studies of flows that can provide new methods for designing more efficient equipment. The article presents a method for solving problems of optimization of hydrodynamic parameters in various devices, which includes two stages. The first stage is the visualization of flows to reveal the structure during the movement of liquids and gases. The second stage is optimization of the geometry of the flow paths based on the identified organization of the flow structure to reduce the resistance of the flow paths and improve the operating parameters of power equipment.

The method of visual diagnostics of the flow structure made it possible to reveal the wave character of the distribution of the pulsation velocity components in the cross section of channels with stable parameters both in space and in time. An odd number of half-waves of transverse structures have a positive effect on an increase in the flow rate at a given pressure, an even number of half-waves increases energy dissipation and other negative phenomena. 


\section{References}

1. Feynman Richard. The_Feynman_Lectures_on_Physics_Vol_I (2012)

2. Loytsyansky L. G. Mechanics of fluid and gas: textbook for universities. Moscow: Bustard, 840 p. (2003)

3. Chashechkin Yu.D., Mitkin V.V. A visual study on flow pattern around the strip moving uniformly in a continuously stratified fluid, J. of Visualization. V. 7. No. 2. P. 127-134. (2004)

4. Prokhorov V.E., Chashechkin Yu.D. Visualization and acoustic sounding of the fine structure of a stratified flow behind a vertical plate, Fluid Dynamics.V. 48. No. 6. P. 722-733. (2013)

5. Van Dyke. An album of fluid motion. / Stanford, California: The Parabolic Press. (1982)

6. Brian J. Cantwell. Organised motion in turbulent flow., Ann. Rev. Fluid Mech. 1981. - Vol. 13. - P. 457-515. (1981)

7. Davletshin I.A. Space Time Structure and Drag in Round Pipe with Periodic Flow Rate Pulsation, Miheev N.I., Romanov D.I..// Proc. 13th Int. Conf. On the Methods of Aerophysical Research. Pt 1. - Novosibirsk: Publ. House "Parallel".- P.88-93 (2007)

8. Arsiry Vasyl, Reconstruction of turbomachines on the basis of the flow structure visual diagnostics ARSIRI Vasyl, KRAVCHENKO Oleg., International Journal Mechanics and Mechanical Engineering, 2018. Volume 22. Number 2, P 405-414 ISSN: 1428-151 1e-ISSN: 2354-0192 (2018)

9. Abdul Rahim A. Khaled. Modelling and Theoretical Analysis of Laminar Flow and Heat Transfer in Various Protruding-Edged Plate Systems, Journal of Electronics Cooling and Thermal Control Vol.5 No.3, September 16, (2015)

10. Nikulin A., Moita A.S., Khliyeva O. Effect of Al2O3 nanoparticles on laminar, transient and turbulent flow of isopropyl alcohol, International Journal of Heat and Mass Transfer. Vol. 130. P. 1032-1044. (2019)

11. Khliyeva O., Ryabikin S., Lukianov N., Zhelezny V. Experimental study of heat exchange and hydrodynamics at the laminar flow of nanocoolant based on propylene glycol and A12O3 nanoparticles, Eastern european journal of enterprise technologies. Vol. 1/8 (85). P. 4-12. (2017)

12. Velikanov M.A. Dynamics of channel flows. Part 1 (Flow Structures) - P.323 (1954) 